\title{
Mécanisme de la mutagenèse SOS chez les bactéries
}

\author{
Raymond Devoret
}

\section{Société Française de Génétique \\ Président \\ A. Nicolas \\ Président d'honneur \\ F. Jacob l'influence que peuvent avoir les radia- tions ou les produits chimiques pré- sents dans notre environnement sur la fréquence d'apparition des cancers. En effet, l'examen des statistiques montre que la majorité des cancers est liée à des atteintes de l'organisme par cer- tains agents physiques, comme le rayonnement ultraviolet solaire, ou cer- tains agents chimiques, comme le ben- zopyrène présent dans la fumée de tabac.}

Vice-président

C. Stoll

Secrétaire général

M. Solignac

Trésorier

P.-M. Sinet

Prière d'adresser toute correspondance au Secrétariat général de la SFG, Michel Solignac, laboratoire de biologie et génétique évolutives, bâtiment 13, Cnrs, 91198 Gif-sur-Yvette Cedex, France.

Comité de rédaction

A. Bernheim

M. Fellous

J. Génermont

F. Minvielle

R. Motta

A. Nicolas

S. Sommer

D. de Vienne

Secrétaire

M.-L. Prunier
On s'inquiète, à juste raison, de

La mutagenèse bactérienne utilisée comme test pour détecter les cancérigènes potentiels

Il y a plus d'une dizaine d'années que des tests de mutagenèse chez des bactéries ont été mis au point pour détecter les cancérigènes potentiels qui menacent notre santé. Ces tests de mutagenèse, rapides et peu coûteux, ont remplacé en partie les tests de production de tumeurs sur les petits mammifères de laboratoire $[1,2]$.

Le cancer étant une maladie propre aux organismes multicellulaires évolués, il peut paraître paradoxal de recourir pour identifier des substances cancérigènes, à des bactéries, qui sont des êtres minuscules situés au bas de l'échelle de l'évolution.

La bactérie et l'éléphant possèdent le même matériel héréditaire

Comme l'a souligné André Lwoff, la nature ne connaît pas de paradoxes mais seulement des solutions. Le paradoxe soulevé plus haut est résolu si l'on admet que la bactérie comme l'éléphant possèdent le même support des caractères héréditaires constitué par de l'ADN.
On sait depuis le début du siècle que c'est en altérant l'ADN, le réceptacle universel des caractères héréditaires, que certains agents physiques et chimiques de notre environnement provoquent des cancers.

Il a été établi que, lorsque l'ADN est endommagé, des lésions subsistent malgré l'existence de processus de réparation. Ce sont ces lésions résiduelles qui sont génératrices de mutations. Lors des dix dernières années, il a été démontré que des mutations peuvent altérer l'activité des oncogènes, soit par action directe, soit par action sur des anti-oncogènes.

Mutagenèse : errare naturale est

L'erreur est dans la nature : le terme de mutagenèse désigne le processus naturel qui engendre les erreurs de reproduction de l'ADN.

L'apparition de mutations dans l'ADN des mammiferes peut conduire à : (1) des maladies héréditaires lorsqu'elles touchent les cellules germinales ; (2) des cancers lorsqu'elles touchent les cellules somatiques; en revanche, il faut aussi considérer que (3) les mutations, filtrées par la sélection naturelle, sont le moteur de l'évolution biologique.

Des plasmides, qui se reproduisent aussi bien dans les cellules de mammifères que chez des bactéries, montrent que les mutations provoquées par les cancérigènes sont de nature semblable dans les deux types d'organismes [3] Cela n'est pas pour nous surprendre, car non seulement l'ADN est le support des gènes, mais les enzymes qui agissent sur l'ADN lors de sa réplication ou de sa réparation ont conservé une grande parenté au cours de l'évolution. 
L'excmple suivant le montre : des bactéries dont l'ADN polymérase a été renduc inactive par mutation survivent grâce à l'implantation, dans leur génome, du gène de la polymérase $\beta$ de souris [4]. Les bactéries greffées peuvent alors se reproduire avec la vitesse d'unc bactéric de type sauvage. Cette "thćrapie génique" donne un exemple de la conservation de la fonctionnalité enzymatique au cours de l'ćvolution, fonctionnalité corroboréc par la structure semblable des domaines catalytiques des enzymes.

Malgré les progrès de la connaissance sur la nature des mutations produites par les cancérigènes, nous ignorons encore le mécanisme qui transforme une lésion de l'ADN en mutation dans les cellules de mammifères. Cette ignorance est d'autant plus criante que l'on avance à pas de géant dans la reconnaissance et la localisation des mutations responsables des maladies héréditaires.

A l'heure actuelle, il cst beaucoup plus aisć de déterminer le mécanisme de la mutagenèse chez les bactérics que chez l'homme. La connaissance acquise chez les bactérics a valcur d'exemple.

\section{Modes d'apparition des mutations}

Qu'est-ce qu'une mutation ?

Un réplicon est une sćquence d'ADN qui sc réplique de façon autonome. Le génome des bactérics constituc un réplicon. Un chromosome de cellule de mammifere est un réplicon.

On définit par le terme de mutation tout changement de séquence d'un réplicon qui est transmissiblc au réplicon fils. Le changement de séquencc cst défini par rapport à un témoin, un standard de référence, dit type sauvage, représenté le plus souvent par la séquence d'ADN de l'organisme le plus représentatif de l'espèce.

\section{Les mutations spontanées}

Les mutations dites spontanécs sont rares car la presque totalité des organismes se reproduisent conformément à leurs parents. Lcs " mutations spontanécs " sont définies par opposition aux "mutations provoquécs ", ces dernières ayant une causc plus immédiatc et le plus souvent connue.
Tout récemment, les principales causcs de la mutagenèse spontanée ont ćtć identifiécs. La mutagenèse spontanćc est due : (1) à des erreurs commises par les cnzymes de la réplication de l'ADN ; (2) à l'introduction frauduleuse, naturelle, d'analogues de bases dans l'ADN.

En répliquant l'ADN, la polymérase fait des erreurs qui peuvent être effacées pendant ou bien immédiatement après le processus de réplication. La réparation des mésappariements est un processus efficace qui corrige l'erreur une fois la rćplication faitc [5].

Le métabolisme aérobie est responsable de mutations spontanćes par introduction frauduleuse de bases nucléiques modifiées dans l'ADN. Certes, l'aérobiose permet aux cellules une meilleure utilisation de l'ćnergie chimique, mais elle engendre aussi de nombreux intermćdiaires métaboliques qui attaquent la moléculc d'ADN. Par exemple, unc fraction significative de la guanine est transforméc en oxyguaninc. I.orsque de l'oxyguaninc est incorporéc dans l'ADN, clle s'apparic à de l'adéninc au lieu de la cytosine et produit unc mutation à la réplication suivante. Lcs mutations par introduction d'oxyguanine dans l'ADN auraient une frćquence cxtrêmement élcvée s'il n'y avait pas au moins trois enzymes pour cmpêcher ou réparer un tel événement délétère [6].

Ames fait l'hypothèse que les lćsions de l'ADN produites par le mćtabolisme aérobie cngendrent une incapacité de réparation croissant avec l'âge qui aboutit au vieillissement [7]. Bref, en vieillissant nous rouillons.

La fréquence des mutations spontanćes est rare parce que l'apparcil enzymatique de la rćplication de l'ADN maintient la plus grande précision possible. Chez les bactéries, la probabilité de mutagenèse est faible, ellc cst ćgale à 10-10 par nuclćotide par réplication ; chez l'homme, ce taux est mille fois moindre. La fidélité de la réplication augmente d'un factcur 1000 en passant des bactérics aux mammifères [8]. Ccla montre que, lors de l'ćvolution, le bricolage génétique a cu pour effet dc pcaufiner la qualité et le nombre des protéines impliquées dans la réplication et la réparation.
Fréquence des mutations induites par les cancérigènes

Les " mutations provoquées " apparaissent avec une fréquence 100 à 1000 fois plus ćlevée que les mutations dites "spontanćcs".

Plus de $85 \%$ des cancérigènes chimiques sont des mutagènes chez les bactéries. Il existe une relation étroite entre le pouvoir cancérigène d'un composé chimique et sa capacité d'endommager l'ADN [9].

Les lésions de l'ADN produites par les cancérigènes déclenchent chez les bactéries trois processus successifs de réparation. Ces processus sont représentatifs de l'existence de fonctions SOS.

\section{Réplication de l'ADN endommagé}

\section{La réplication bidirectionnelle}

Il faut rappcler que : (1) le chromosome bactérien est un cercle qui possède unc origine de réplication ; et que (2) la duplication s'opère de façon bidirectionnelle. Deux fourches de réplication de sens opposé se forment à partir de l'originc, progressent le long de l'ADN pour se rejoindre au terminus du chromosome, situć de façon diamćtralement opposćc à l'originc.

Dans une culture bactérienne en croissance dans laquclle les cellules ne sont pas synchrones, on peut calculer qu'en moyenne les chromosomes sont déjà dupliqués sur $63 \%$ de leur longucur. On voit qu'en cas d'endommagement du chromosome, les gènes proches de l'origine ont un avantage sélectif. Comme ils sont dupliqués les premicrs, ils sont plus facilcment réparés par recombinaison que les gènes situés près du terminus de la réplication. On constate d'ailleurs que les gènes essenticls se trouvent près de l'origine de réplication. Les gènes situés près du terminus du chromosome pcuvent être perdus sans dommage fonctionncl grave pour la bactéric.

On sait que l'apparcil qui effectuc la réplication est complexe. La réplicase cst composée de sept protéines qui peuvent être temporaircment associćcs à d'autres. C'est pourquoi, dans ce bref exposé, j’utiliscrai pour qualifier le complexc de réplication le terme de rćplisome. 
Rencontrant une lésion, le réplisome joue à saute-mouton

Si le réplisome aborde une lésion avant qu'elle n'ait pu être réparée, il bute sur la lésion, stoppe sa progression et se décroche de l'ADN. Le réplisome va reprendre la synthèse du brin complémentaire à environ $1 \mathrm{~kb}$ en aval de la lésion [10]. La structure formée est celle d'un ADN double brin présentant une discontinuité faite d'un simple brin.

La structure ainsi engendrée (figures 2, $3 a$, 4a) est désignée en anglais par le terme imagé de gapped $D N A$. On pourrait dire que, lorsqu'il rencontrc une lésion, le réplisome ouvre et referme une parenthc̀se vide d' $1 \mathrm{~kb}$.

La structure décrite ci-dessus est intéressantc à plus d'un titre.

\section{Le signal SOS}

L'ADN simple brin formé attire la protéine RccA, qui s'active en coprotéasc pour cliver le répresseur LexA (figure 2) et ainsi déclencher la série des trois processus de réparation et de mutagenèse $[11,12]$.

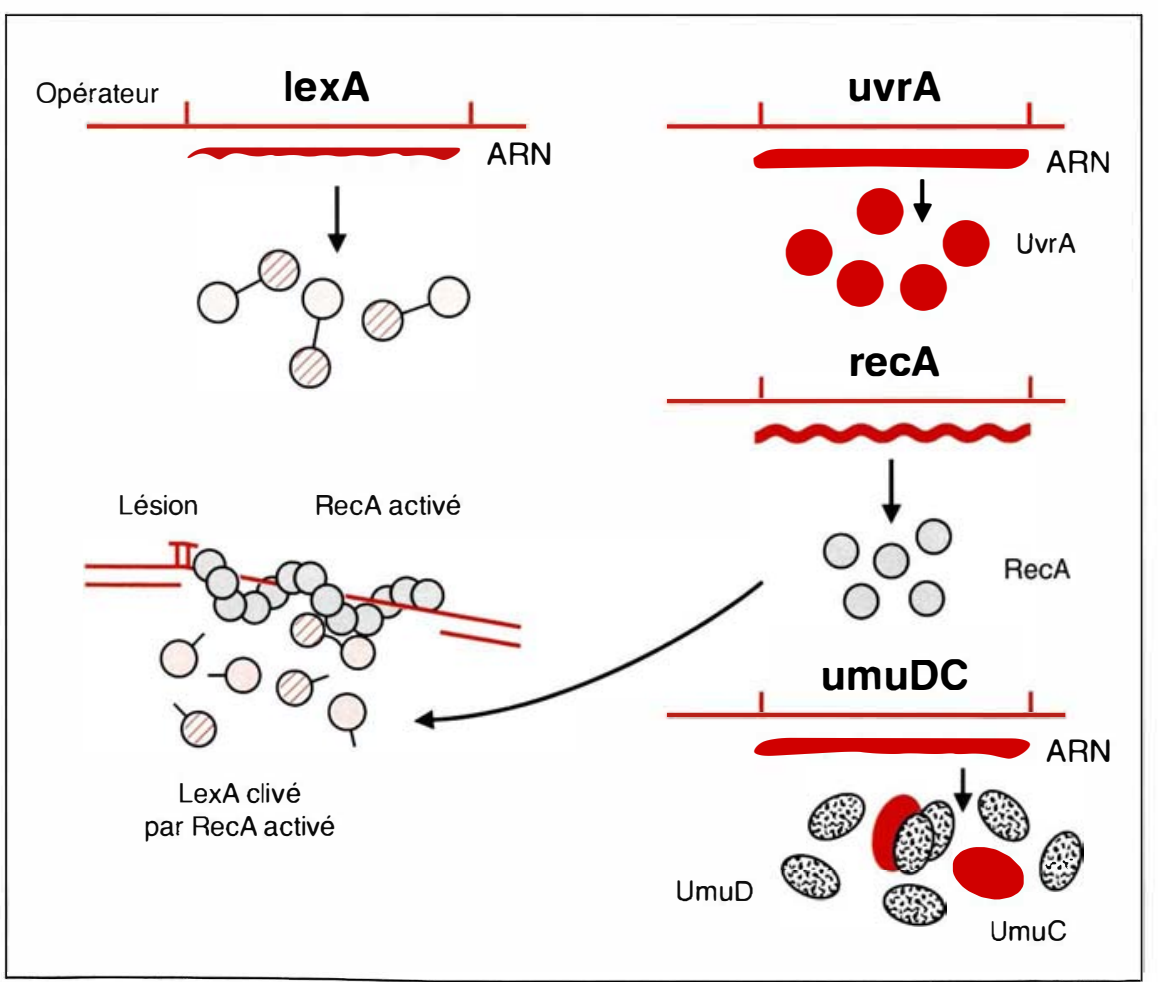

$m / s n^{\bullet} 3$, vol. 9 , mars 93

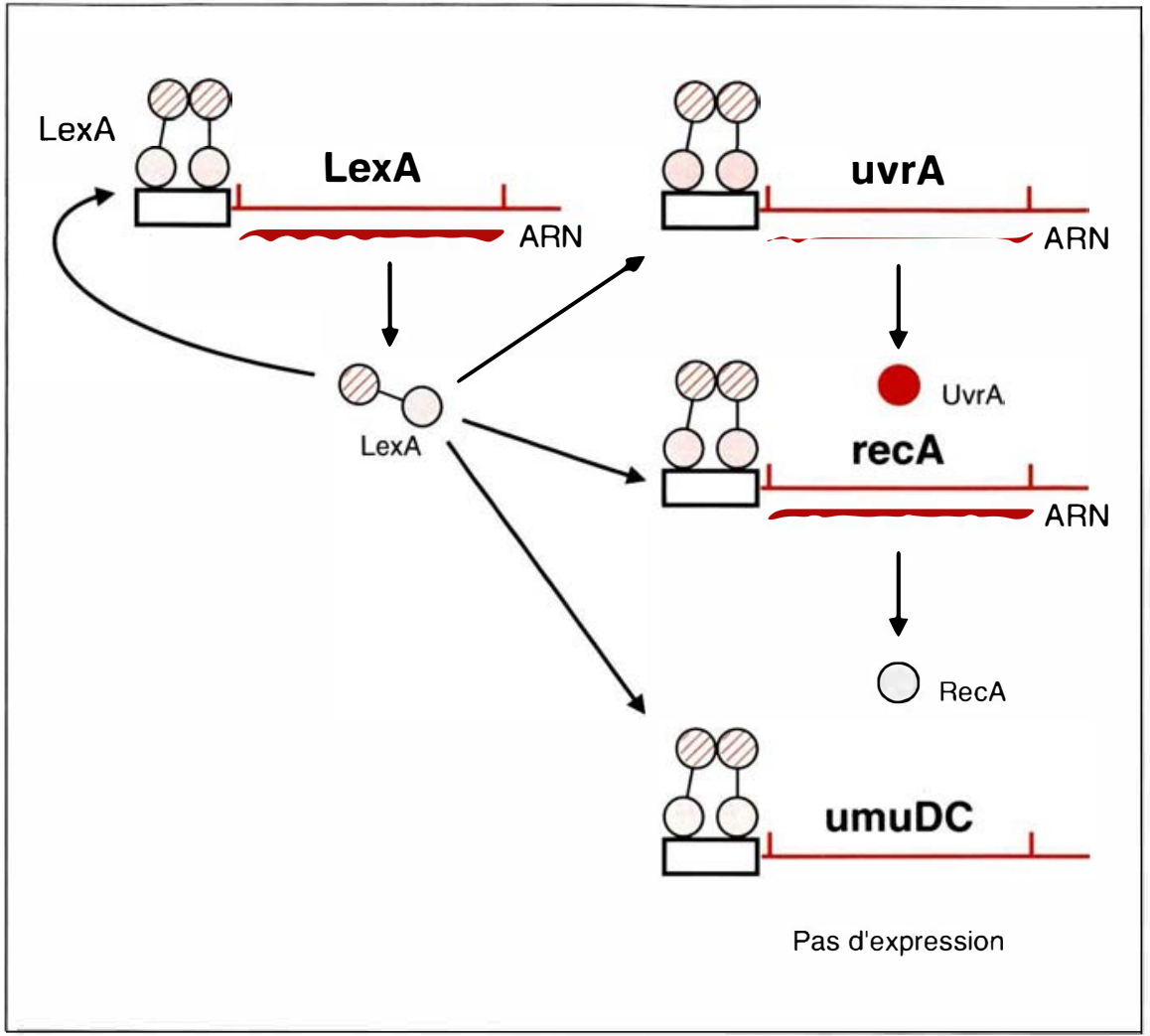

Figure 1. Répression des gènes SOS (état physiologique). Le répresseur LexA (petite haltère) est fixé au site opérateur-promoteur du gène LexA et des autres gènes SOS, et réprime leur transcription. La protéine RecA est représentée, par des cercles gris et la protéine UvrA, par des cercles rouges.
Figure 2. Dérépression des gènes sos létat induit après lésions de I'ADN). La protéine RecA forme un filament hélicoidal sur l'ADN simple brin engendré par l'arrêt local de la réplication qui introduit une discontinuité dans la double hélice. Le répresseur LexA (haltères) s'attache au complexe ADNsimple brin-RecA sur lequel il est clivé. L'inactivation du répresseur LexA par clivage entraîne, entre autres: (1) la synthèse de la protéine UvrA protéine impliquée dans la réparation par excision); (2) la synthèse de la protéine RecA (chapelet hélicoïdal); (3) la synthèse des protéines mutagènes UmuD (ellipsoïde tacheté) et UmuC (ellipsoïde rouge). 
Le complexe ADN simple brinprotéine RecA, en provoquant l'inactivation de la protéine LexA, constitue un signal SOS, inducteur des 20 gènes SOS (figure 2). Dans des conditions optimales de croissance bactérienne, les gènes SOS sont réprimés, ils n'expriment pas, ou bien peu, les protéines pour lesquelles ils codent [11].

Lorsque le répresseur LexA est clivé, les gènes SOS, impliqués dans les divers processus de réparation, sont exprimés avec une cinétique propre qui assure le déroulement séquentiel des processus [13].

Il faut souligner qu'in vivo, un simple brin d'ADN n'est jamais nu. Il est soit recouvert par la protéine SSB (singlestrand binding protein) ou, comme c'est le cas à la suite de lésions, il est recouvert par la protéine RecA.

Il serait satisfaisant que les lésions ellesmêmes déclenchent l'activation de la protéine RecA et donc l'induction des fonctions SOS [14]. Mais la protéine RecA est activée non pas par une lésion spécifique mais par la discontinuité de la double hélice due à la présence d'une lésion.

Le signal SOS : un substrat pour la réparation

Le complexe ADN simple brinprotéine RecA constitue un substrat qui permet d'apparier le simple brin avec un brin homologue, première étape de la recombinaison induite (figure $3 b$ ).

Le même substrat, s'il n'a pas été impliqué dans un processus de recombinaison homologue, doit être réparé. La discontinuité de la double hélice avec la persistance d'un simple brin entraîne la mort du chromosome. Le processus de mutagenèse SOS est capable de restaurer la structure double brin de l'ADN (figure 4).

Les fonctions SOS s'éteignent lorsque la réparation restaure la structure de la double hélice et fait ainsi disparaître le signal SOS.

\section{Les processus de réparation SOS}

Nous allons brièvement décrire dans leur principe les trois systèmes de réparation SOS : excision, recombinaison,

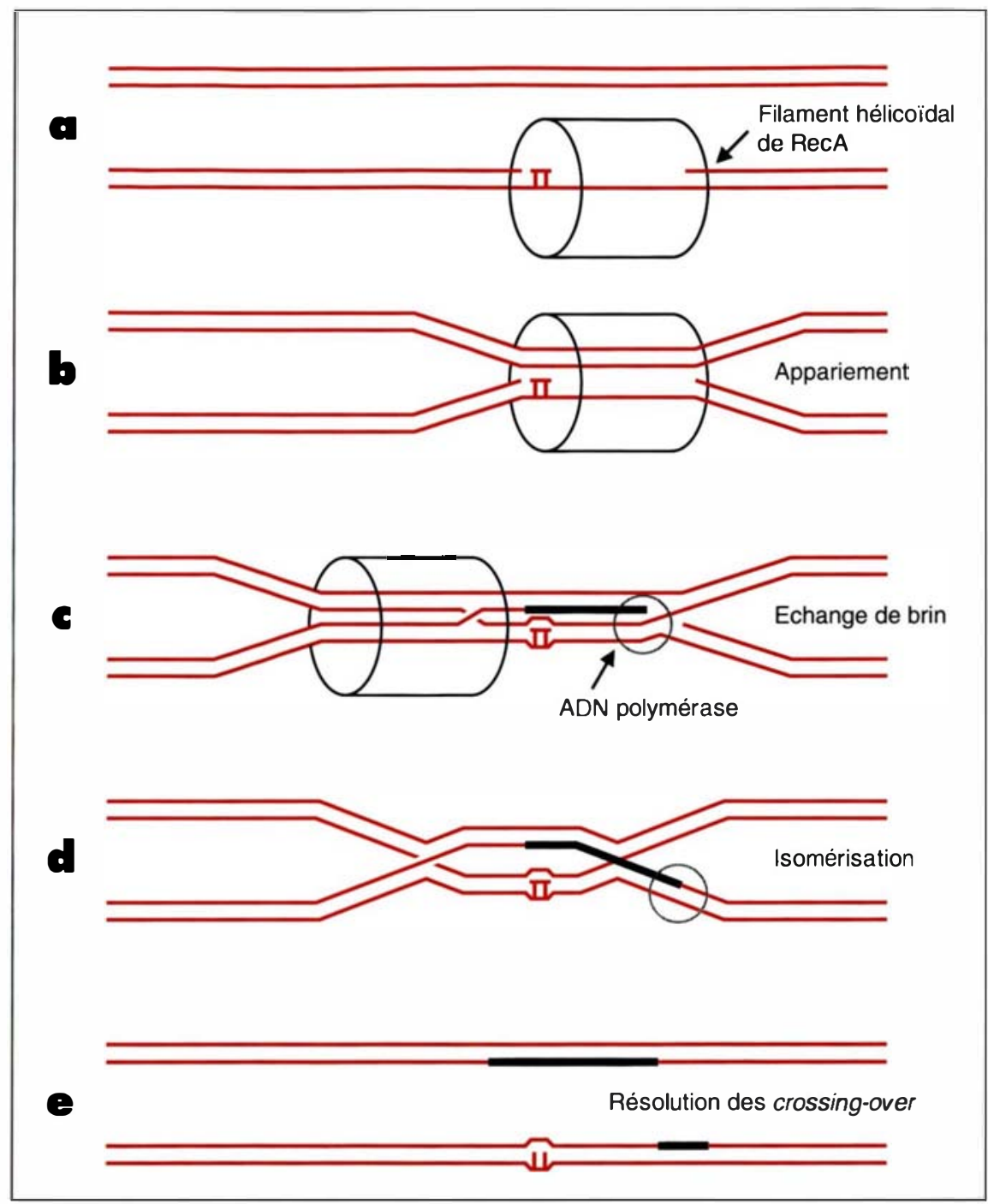

Figure 3. Réparation par recombinaison. La lésion de l'ADN est représentée par un dimère de thymine qui peut entrer dans un processus de recombinaison réparateur. A droite du dimère de thymine (a) se forme une discontinuité dans la double hélice par arrêt de la réplication du brin fils; la protéine RecA forme un filament protecteur de l'ADN simple-brin (cylindre) et favorise l'accolement d'un brin opposé pour restaurer la double hélice (b). II y a synthèse en trans pour remplacer le brin emprunté (c). II se forme une jonction de Holliday (d) qui est résolue en (e). La coupure de la jonction aboutit à rétablir la structure normale de I'ADN. On voit les portions d'ADN échangées et resynthétisées (traits épais). (Schéma de Paul Howard-Flanders, communication personnelle).

réplication infidèle, gouvernés par l'expression, entre autres, des gènes $u v r A, \operatorname{rec} A$, et umuDC (figures 1 et 2) [15]. Ces gènes sont soumis à un régulateur commun, le répresseur LexA.

La réparation par excision tend à éliminer la lésion. Le processus excise 11 nucléotides sur le brin endommagé (cinq nucléotides en amont et cinq en aval du nucléotide endommagé). Les 11 nucléotides sont resynthétisés par réplication locale. L'efficacité de la réparation par excision n'est que de $85 \%$. Après la réparation par excision, il reste encore $15 \%$ de lésions dans l'ADN [16].

La réparation par recombinaison tend 
à mettre de côté les lésions qui n'ont pu être excisées. Le principe de la réparation par recombinaison est simple : si une cellule possède un jeu dc deux chromosomes, la probabilité qu'une lésion affecte précisément le même nucléotide sur chaque chromosome est extrêmement faible. La réparation par recombinaison consiste à prélever un brin d'ADN de la région non endommagée d'un chromosome homologue pour remplacer la portion de brin altéré. Le chromosome donneur est lui aussi restauré dans sa séquence originale par une simple resynthèse locale (figure $3 c$ ).

Il faut noter qu'après la réparation par recombinaison, un seul brin sur quatre reste porteur de lésion. Il n'y a pas de risque de perte d'information car, dans la double hélice endommagée, le brin sain pourra être dupliqué, il sera conforme à la séquence d'ADN originalc.

La réparation par recombinaison a une efficacité qui se situe entre 50 ct $65 \%$. Après le passage de deux processus de réparation par excision et par recombinaison, il reste environ 5 à $7 \%$ de lésions non réparées [17].

Il faut souligner que l'excision et la recombinaison sont deux processus de réparation fidèles qui n'introduisent pas de mutations dans l'ADN.

Le processus majeur qui transforme une lésion en mutation va être décrit ci-après.

\section{La mutagenèse SOS}

La réplication fautive : la mutation est en face de la lésion

La réparation fautive va répliquer l'ADN porteur de lésion qui présente une discontinuité de sa double hélice. Pour ce faire, la réplicase va ignorer la lésion se trouvant sur le brin père et va synthétiser le brin fils en face de la lésion. La réplicase perd alors la fidélité qui la caractérise. La réplicase est " désaccordée ", elle met une base nucléique en face de la lésion résiduelle (figure 4c). La réplication fidèle est temporairement suspendue pour restaurer un réplicon viable, même si c'est au prix d'une altération du code génétique. La réplication fautive répare $m / s n^{\circ} 3$, vol. 9, mars 93

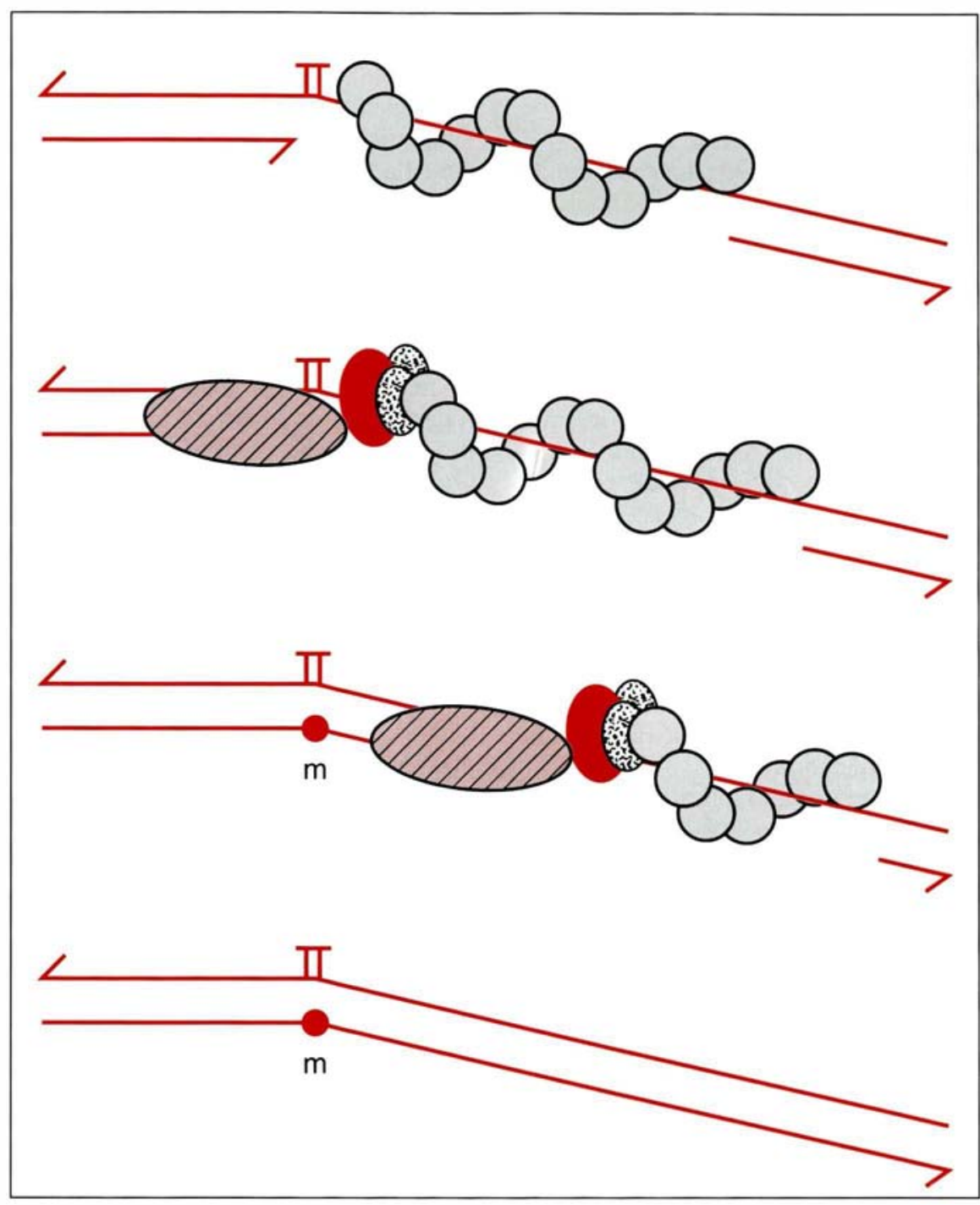

Figure 4. Réparation fautive induite. L'ADN simple brin enrobé par la protéine RecA peut entrer dans un processus de réplication fautive après la réparation par recombinaison. Pour cela, il faut que le complexe Umu ${ }^{\prime}{ }_{2} C$ soit formé après clivage de la protéine UmuD en UmuD' (croissants tachetés). Alors se déroulent les processus suivants : le complexe Umu ${ }^{\prime}{ }_{2} C$ agit vers la droite en repoussant le filament de RecA qui se raccourcit, il s'arrime à gauche à la polymérase pour lui permettre de franchir la lésion. Le symbole " $m$ " désigne la mutation produite, I'ADN-polymérase est indiquée par un ovale, et le complexe Umu ${ }^{\prime}{ }_{2}-U m u C$ est modifié par rapport au complexe $U m u D_{2}-U m u C$ présent dans la figure 2.

$50 \%$ des lésions qui ont échappé à la réparation par excision et par recombinaison.

Il faut souligner que la réparation fautive produit une mutation non pas sur le brin qui porte la lésion mais en face sur le brin d'ADN fils.

Une lésion de l'ADN n'est pas une mutation. En outre, une mutation n'est détectée que dans un organisme qui a pu se reproduire, que ce soit un minuscule virus ou un être humain.

Chronologie des trois processus de réparation

$\mathrm{Si}$ tous les gènes $\mathrm{SOS}$ sont réprimés 
par lc répresscur LcxA, comment peuton obtenir des processus successifs dc réparation?

La cinétique des trois processus de réparation décrits plus haut est fondéc sur l'affinité différente du répresscur LexA pour chacun des opératcurs des gènes SOS. Si les opératcurs des gènes qui gouvcrnent la réparation par cxcision, uvrA par exemple, ont une affinité moins grande pour le répresseur LexA, il est clair qu'unc diminution minime du répresseur LexA va produire unc dérépression rapide [18]. C'est pourquoi la réparation par excision va être la plus précoce et durer 20 minutes après l'apparition des lésions. La réparation par recombinaison néccssite un nivcau élevé de la protéinc RecA, qui cst atteint au bout dc 40 minutes. RecA a le temps de former de longs filaments entourant le simple brin produit par la discontinuité de la réplication.

La réparation par réplication fautive va être la dernière à être mise cn œuvrc car clle néccssitc, dans un premier tcmps, unc synthèse élcvéc de protéines UmuD et UmuC (figures 2 et 4). Dans un deuxic̀me temps, la protéine RecA doit effectucr la maturation de la protéinc UmuD en UmuD' pour donner le complexe mutagène UmuD', ${ }_{2}$ [19-21]. Le temps nécessairc à la synthèse ct à la maturation du complexe UmuD', ${ }_{2} \mathrm{C}$ est dc 60 minutes [22]. Une fois le complexc UmuD' ${ }_{2} \mathrm{C}$ formé, il permet, lorsqu'il cst présent au niveau de la lésion, de modifier la fidélité avec laquelle lc réplisome effectuc la duplication de brin (figure 4c).

La chronologie de formation du complexe UmuD' ${ }_{2} \mathrm{C}$ fait que le processus de réplication fautive se déclenche après que le processus de recombinaison a pu sc réaliser.

\section{Conclusions}

Le déroulement séquenticl des fonctions SOS

Il est orchestré par une hiérarchie de régulations. Lc système de mutagenèsc implique des étapes successives contrôlécs car la mutagenèse cst un proces-
l'ADN de muter n'importe où et n'importe comment. La mutagenèse est seulement misc en ouvre comme ultime recours pour éviter la disparition d'un réplicon endommagé par des lésions.

\section{Des fonctions SOS dans les cellules de mammiferes ?}

Des systèmes de réparation analogues chez les bactéries et dans les cellules de mammifères ont été découvcrts. La réparation par cxcision des lésions se produit de façon semblable.

On sait que l'exposition des cellules aux rayons ultraviolets du soleil provoque la formation de dimc̀res de pyrimidine $\mathrm{T} \Lambda \mathrm{T}(60 \%), \mathrm{T} \Lambda \mathrm{C}(30 \%)$ ou $\mathrm{C} \Lambda \mathrm{C}(10 \%)(\mathrm{T}=$ thyminc, $\mathrm{C}=$ cytosine). Chez l'homme, l'cxcision des lésions fait partie des proccssus majeurs de réparation de l'ADN [23]. Les cnfants atteints de Xeroderma pigmentosum, une maladie qui les rend incapables de réparcr l'ADN par excision, vivent un drame permanent du fait de la préscnce de rayons ultraviolets dans la lumière solaire. Les gènes et lcs protéines qui assurcnt la réparation par cxcision chez l'homme commencent à être identifiés [23].

Existe-t-il chez les mammifères des fonctions SOS contrôlécs de façon scmblablc à cclles trouvées chez les bactéries?

Sarasin et Hanawalt [24] ont montré que le virus SV40 dont l'ADN a été cndommagé par les ultraviolets a unc meilleure survie s'il se reproduit dans des ccllules de rein de singc préalablement irradiées. Des mutations apparaissent dans la descendance du virus ; clles sont situées, avec un décalage de base, en face des lésions dans le brin d'ADN complémentairc néosynthétisé. Ce phénomène ressemble à la réactivation du phage dans unc bactérie cndommagée par les ultraviolets $\lfloor 17$, 25]. L'existence d'un tcl phénomène inductible ct mutagènc dans les cellules de mammifères cst une preuve indirecte en favcur de l'existence de fonctions scmblables aux fonctions induites par des lésions de l'ADN.

La protéine Rad51 de la levure Saccharomyces cerevisiae possède unc grande homologic de séquence (environ $55 \%$ ) avec cellc de la protéine RecA d'E. coli [26]. Un autre analogue de la protéine
RecA vient d'être identifié dans les cellules de mammifères (91\% d'homologic avec Rad51) (Ogawa, communication personnclle).

Du fait de la complexité de la chromatine qui enveloppe l'ADN des cellules de mammifères, il doit exister un processus d'ouverture ct de fermeture de la chromatine qui vient compliquer les processus de réparation des lésions. On peut supposer que la protéine RecA, qui joue un rôle esscntiel de plaque tournante dans lcs fonctions SOS bactériennes, peut avoir évolué dc tellc sorte quc, dans les ccllules de mammitères, clle gouverne les fonctions d'un "réparosome " bicn plus complexc [27]

R. Devorct, groupe d'étude "Mutagenèse et Cancérogenèse ", laboratoirc d'enzymologic du Cnrs, 91198 Gif-sur-Yvettc, France.

\section{Remerciements}

L'auteur est reconnaissant à Mélanic Pierre pour son aide dans la réalisation des dessins et la misc en place des références, à Adriana Bailone et Suzanne Sommer pour leur correction du manuscrit.

Ce travail a été soutenu par des subventions de la Communauté européenne $\mathrm{n}^{\circ}$ BI6-E-145-F, de l'Association pour la recherche sur lc cancer $\mathrm{n}^{\circ} 3693 \mathrm{ct}$ de la Fondation pour la recherche médicale.

\section{Summary}

Mechanism of SOS mutagenesis in bacteria

DNA is damaged by physical or chemical carcinogens present in our cnvironment or produced by metabolism. DNA damage is a stumbling block for the replisome (the replication apparatus) and may lead to cell death. Yet, many cells survive because of the induction of SOS functions that control threc successive repair processes: excision, recombination, induciblc errorprone repair. The third repair process is mutagenic. It accounts for the mechanism of the mutations produced by carcinogens. 


\section{Références}

1. Devoret R. Des tests bactéricns pour identificr les cancérigènes potenticls. Pour la Sciense $1979 ; 24: 62$

2. Hofnung M. Progrès dans la détection des cancérigèncs ct des mutagèncs : lc SOS chromotest. Biofutur 1983; 45.

3. Sarasin A. SOS responsc in mammalian cells. Cancer Invest 1985; 3 : 163.

4. Swcasy JB, Loeb LA. Mammalian DNA polymerase beta can substitute for DNA polymerase I during DNA replication in E. coli. $J$ Biol Chem 1992 ; 267 : 1407-10

5. Radman M, Wagner R. The high fidelity of DNA duplication. Sci Am 1988 ; ? : 24-30.

6. Horiuchi ' $\Gamma$, Maki H, Sckiguchi M. Mutators and fidclity of INA replication. Ann Inst Pasteur $1989 ; 87$ : 309-36.

7. Fraga CG, Shigenaga MK, Park JW, Degan P, Ames BN. Oxidative damage to DNA during aging : 8-hydroxy-2'deoxyguanosine in rat organ DNA and urinc. Proc Natl Acad Sai USA 1990; 87 : 4533-7.

8. Drakc JW. Mutation rates. Bio Essays $1992 ; 14$ : 137-40.

9. McCann J, Choi E, Yamazaki E, Ames BN. Detection of carcinogens as mutagens in the Salmonella/microsome test : Assay of 300 chcmicals. Proc Natl Acad Sci USA $1975 ; 72$ : 5135-9.

10. Howard-Flanders P. DNA repair and recombination. Br Med Bull 1973; 29 : 226.

11. Robcrts JW, Devoret R. Lysogenic induction. In : Hendrix RW, Roberts JW, Stahl FW, Wcisbcrg RA, eds. Lambda II chap- ter VII. Cold Spring Harbor: Cold Spring Harbor Laboratory 1983 : 123-44.

12. Sassanfar M, Robcrts JW. Nature of the SOS inducing signal in $E$. coli : the involvement of DNA replication. J Mol Biol 1990 ; $212: 79$.

13. Little JW, Mount DW. The SOS regulatory system of $E$. coli. Cell $1982 ; 29: 11$.

14. Lu C, Scheucrmann RH, Echols H. Capacity of RecA protcin to bind preferentially to UV lesions and inhibit the editing subunit epsilon of DNA polymerase III : a possible mechanism for SOS-induced targeted mutagencsis. Proc Natl Acad Sa USA 1986 ; $83: 619$

15. Kenyon CJ, Walker GC. DNA-damaging agents stimulate gene expression at specific loci in Escherichia coli. Proc Natl Acad Sa USA 1980 ; 77 : 2819.

16. Lin JJ, Sancar A. (A)BC cxonuclease : the $E$. coli nuclcotide excision repair enzyme. Mol Microb 1992; 6 : 2219-24.

17. Devorct R, Blanco M, George J, Radman M. Recovery of phage lambda from ultraviolet damage. In : Hanawalt PC, Sctlow RB, eds. Molecular Mechanisms for Repair of DNA, part A. New York : Plenum Publishing Corporation, $1975 ; 155-71$.

18. Bertrand-Burggraf E, Hurstel S, Daunc M, Schnarr M. Promoter propertics and negative regulation of the uvrA gene by the LexA repressor and its amino-terminal DNA binding domain. J Mol Biol 1987 ; 193 : 293.

19. Nohmi T, Battista JR, Dodson LA, Walker GC. Rec-A-mediated cleavage activates UmuD for mutagencsis : mechanistic relationship between transcriptional derepression and posttranslational activation. Proc Natl Acad Sci USA 1988 ; 85 : 1816.
20. Burckhardt SE, Woodgate R, Scheucrmann RH, Echols H. UmuD mutagenesis protein of Escherichia coli : ovcrproduction, purification and clcavage by RecA. Proc Natl Acad Si USA 1988 ; 85 : 1811.

21. Shinagawa $H$, Iwasaki $H$, Kato $T$, Nakata A. RecA protein-dependent cleavage of UmuD protcin and SOS mutagenesis. Proc Natl Acad Sci USA 1988; 85 : 1806.

22. Bailone A, Sommer S, Knczevic J, Dutreix M, Devoret R. A RecA protein mutant deficient in its interaction with the UmuDC complex. Biochimie 1991；73：479.

23. Wceda G, Van Ham RCA, Vermculen W, Bootsma D, Van Der Eb AJ, Hocijmakers JH. A presumed DNA helicase encoded by ERCC-3 is involved in the human repair disordcrs Xeroderne pigmentosum and Cockayne's syndromc. Cell 1990; 62 : 777-91.

24. Sarasin A, Hanawalt P. Carcinogens enhance survival of UV-irradiated simian virus 40 in treated monkey kidney cells : induction of a rccovcry pathway? Proc Natl Acad Sa USA $1987 ; 75: 346$

25. Gcorge J, Devoret R, Radman M. Indirect ultraviolet-rcactivation of phage lambda. Proc Natl Acad Sci U.SA 1974; 71 : 144

26. Abousckhra A, Adjiri A, Fabre F. Scmidominant suppressors pf srs-2 helicasc mutations of Saccharomyces cerevisiae map in the rad51 gene whose sequence predicts a protein with similaritics to procaryotic RecA protcins. Mol Cell Biol 1992 ; 12 : 3224-34

27. Devorct R. Ies fonctions SOS ou comment les bactéries survivent aux lésions de lcur ADN. Ann Inst Pasteur/Actualités 1992; 1 : 11-20.

TIRÉS A PART

R. Devorc

\section{INFORMATIONS SFG}

\section{Résultats des élections du mercredi 20 janvier 1993}

- A l'issue des élections (14 postes étaient à pourvoir), le Conseil Axe 5 - Génétique écologique et des populations : Catherine est ainsi composé :

Axe 1 - Génétique moléculaire et cellulaire: Philippe Benech, Moni- Axe 6 - Génétique quantitative et sélection : André Bervillé, Claude que Bolotin-Fukhara, Pierre Netter, Bernard Perbal, Hubert Pinon, Gaillardin, Frédéric Galacteros, Louis Ollivier. Suzanne Sommer.

Axe 2 - Génétique microbienne : Bénédicte Michel, Alain Nicolas, Pierre Thuriawx.

Axe 3 - Cytogénétique : Roland Berger, Paul Popescu, Gérard Tachdjian. Briand, Claudie Isnard.

Axe 7 - Génétique humaine normale et pathologique : Nicole Baumann, Marie-Paule Lefranc, Pierre-Marie Sinet, Claude Stoll (le nom des nouveaux élus est en italique)

Bureau - Président : Alain Nicolas - Secrétaire général : Michel SoliBriand, Claudie Isnard. 


\section{INFORMATIONS SFG}

\section{Réunions en France}

- La levure : modèle et outil, Gif-sur-Yvette, 25-26 novembre tées sous forme d'exposés (15 min) ou d'affiches. 1993. Ce colloque, organisé par Pierre Netior (CNRS - Centre Pour toute information : s'adresser à P. Notier, CNRS, Centre de Génétique Moléculaire) prolongera une première conférence de Génétique Moléculaire 91190 Gif-sur-Yvette, Fax : 93 (1) organisée à Bombarinos en 1985 (150 participants) et une seconde 09.07.53.22 - Télex : 33 (1) 603578 F

à l'Institut Pasteur de Paris (sous l'égide de la Société Française • $2^{\mathbf{e}}$ réunion "Éléments transposables ", Lyon, 5-7 juillet 1993. de Génétique) en Mars 1991 (350 participants) rassemblant la com- Cette réunion, qui fera suite à celle de Gif-sur-Yvette, se veut munauté des chercheurs d'expression française utilisant la levure d'abord un lieu de rencontres et de discussions, et souhaite poucomme modèle biologique de la cellule euraryolique, comme outil voir fournir à de nombreux jeunes l'occasion d'exposer leurs résuldans l'étude d'autres modèles, ou pour des applications biotech- tats. Thèmes envisagés : Éléments transposables et évolution nologiques. L'accent sera mis surla confrontation entre les diffé- Régulation de la transcription - Mécanismes de contrôle du nomrentes disciplines constituant la biologic cellulairc (biochimie, cyto- bre de copies - Modélisation - Transferts horizontaux - Transpologie, génétique), la diffusion du techniques expérimentales nou- sons et rétrovirus, etc.

velles, l'ouverture européenne (Belgique, Suisse, EMBL à Heldel- Pour tout renseignement : s'adresser à Christian Biémont, bâtiment 403, berg) et la participation des jeunes équipes. Le colloque est ouvert URA Cnrs 243, université Claude-Bemard - Lyon 1, 43, boulevard du à tous les scientifiques du secteur public ou industriel. Les com- 11-Novembre 1918, 69622 Villeurbanne Cedex. Tél. : (16) 72.44.81.98 munications seront examinées par comité scientifique, et présen- — Fax : (16) 78.89.27.19.

\section{Prix Jeanne Loubaresse-Institut Curie 1993}

- Ce prix récompensera un chercheur pour unc découverte effec- Montant du prix attribué au titre de l'année 1993 : 400000 francs. tuéc dans le domaine de la physique, de la chimie, de la biologie, Règlement et formulairc de candidature peuvent ĉtre obtenus à de la clinique ou de l'épidémiologie, découverte susceptible de con- l'adresse suivante

tribuer - à court, moyen ou long terme - à améliorer le dia- Institut Curie - Présidence, Prix Jeanne Loubaresse-Institut Curie gnostic, le traitement ou la prévention du cancer. Cette découverte devra avoir été effectuée dans un laboratoire de recherche public ou privé, implanté dans l'un des douze pays de la Communauté européenne.

1993, 26, rue d'Ulm, 75231 Paris Cedex 05.

Date limite d'envoi du dossier de candidature : lundi 3 mai 1993 (le cachet de La Poste faisant foi).

\section{SOCIÉTÉ FRANCCAISE DE GÉNÉTIQUE DEMANDE D'ADHÉSION}

Cette demande est à adresser au secrétaire général, Michel Solignac, laboratoire de biologie et génétique évolutives, bâtiment 13, Cnrs, 91198 Gif-sur-Yvette Cedex. (Conformément aux statuts, elle sera soumise à l'approbation du bureau lors de sa prochaine séance.)

NOM (Madame, Mademoiselle, Monsieur) :

PRÉNOM :

DATE DE NAISSANCE :

NATIONALITÉ :

FONCTION ET GRADE :

ORGANISME DE RATTACHEMENT:

TITRES ET DIPLÔMES :

ADRESSE PERSONNELLE* (adresse, téléphone) :

ADRESSE PROFESSIONNELLE* (centre ou université, laboratoire, adresse, téléphone, fax) :

DATE :

SIGNATURE :

Montant de la cotisation 1993 : $260 \mathrm{~F}$

Pour les étudiants (avantage financier limité à l'année d'adhésion) : $150 \mathrm{~F}$ par chèque bancaire ou postal libellé à l'ordre de la SOCIÉTÉ FRANÇAISE DE GÉNÉTIQUE. Joindre la photocopie de la carte d'étudiant.

"Marquer d'une croix l'adresse professionnelle ou personnelle où doit être envoyé le courrier. 